\title{
Active Vibration Isolation Using an Electrical Damper or an Electrical Dynamic Absorber
}

\author{
Sang-Myeong Kim, Stanislaw Pietrzko, and Michael J. Brennan
}

\begin{abstract}
This paper describes a theoretical and experimental study to show how an electrical damper or an electrical dynamic absorber, implemented using an electromagnetic actuator and an accelerometer, can control vibration transmission through a vibration isolator. The electrical damper is realized by feeding back the equipment velocity to the actuator with constant gain. The electrical dynamic absorber is realized by feeding back the equipment acceleration through a second-order low-pass filter. Because it is found that the plant on a flexible base is asymptotically similar to that on a rigid base, the optimal parameters of the control filter are determined analytically, independent of the base dynamics. Experimental results show that the electrical dynamic absorber has a similar performance to the electrical damper. The maximum reduction in transmitted vibration achieved was about $38 \mathrm{~dB}$ for both methods. It is also shown that the electrical dynamic absorber is more robust to undesirable dynamics outside the control bandwidth. Another advantage of the electrical dynamic absorber is that it does not require an integrator to transform acceleration into velocity.
\end{abstract}

Index Terms-Acceleration-position feedback (APF), direct velocity feedback (DVFB), electrical damper, electrical dynamic absorber, vibration control.

\section{INTRODUCTION}

W HEN a mechanical structure undergoes severe resonant vibrations, it is common practice to attach dampers and dynamic absorbers to the structure [1]. Dampers are power-dissipating elements that add damping to a structure over a widerange of frequencies. Dynamic absorbers are effectively tuned narrow-band damping devices used to control resonant vibration of a structure. Although the damper and the dynamic absorber are mechanically quite different, both can be equally effective in reducing resonant vibrations. In practice, however, one may be preferred to the other or both may be inapplicable due to difficulties in mechanical construction, design constraints, etc.

Efforts have been made to realize dampers and dynamic absorbers electrically. An example of an electrical damper is an actuator collocated with a sensor at one end and grounded at the other (i.e., grounded actuator installation), together with direct velocity feedback control [2]. Another example is the electrical implemen-

Manuscript received March 22, 2006; revised August 30, 2006. Manuscript received in final form May 1, 2007. Recommended by Associate Editor P. Meckl.

S.-M. Kim is with the EMPA-Materials Science and Technology, Laboratory for Acoustics, Duebendorf, $\mathrm{CH}-8600$, Switzerland, and also with the Phonak Hearing Systems AG, Staefa, CH-8712, Switzerland.

S. Pietrzko is with the EMPA-Materials Science and Technology, Laboratory for Acoustics, Duebendorf, CH-8600, Switzerland.

M. J. Brennan is with the Institute of Sound and Vibration Research, University of Southampton, Southampton SO17 1BJ, U.K. (e-mail: mjb@isvr.soton.ac.uk).

Color versions of one or more of the figures in this paper are available online at http://ieeexplore.ieee.org.

Digital Object Identifier 10.1109/TCST.2007.903376 tation of a skyhook damper for vibration isolation, where an actuator is collocated with a sensor attached to a piece of equipment at one end and supported by a base structure at the other (i.e., structure-borne actuator installation) [3]. For a true skyhook damper to be realized, the base motion should not be affected by the vibration induced by the actuator (i.e., a weakly coupled base). In such cases, structure-borne actuators can be regarded as grounded and the electrical dampers are exactly equivalent to mechanical dampers. The control system is therefore unconditionally stable. Kim et al. [4], [5] recently investigated a general case, where four independent skyhook dampers were applied to each of four mounts connecting a piece of 3-D equipment and a strongly coupled base structure. They showed that the control mechanism and stability of this general case are not quite the same as those discussed in [3]. Experimental results demonstrated that an electrical damper can be realized only within a limited bandwidth in practice. At very low and very high frequencies outside this bandwidth, the control system became unstable for high gain. The objective of the work reported in this paper is to develop a practical controller that is more robust to undesirable dynamics outside the control bandwidth. An electrical dynamic absorber may be suitable for this purpose.

Examples of electrical dynamic absorbers include collocated acceleration feedback controllers [6], [7] and shunt circuits using piezoelectric or electromagnetic actuators [8], [9], where the former are active absorbers requiring external power sources while the latter are passive. Although the authors of [6] and [7] did not mention it explicitly, they employed the principle of the dynamic absorber. These active and passive electrical absorbers are equivalent to mechanical absorbers and thus in theory always stable. In a broad sense, positive position feedback control may fall in this category in that it also uses a resonant circuit to control resonant vibrations [10]. All of these various absorbers were used to control general flexible structures. In this paper, an electrical dynamic absorber is used specifically in a vibration isolation system, where there is a structure-borne actuator and a strongly coupled base structure.

The aim of this paper, therefore, is to investigate the way in which an electromagnetic actuator installed in parallel with a mount may be used as an electrical damper or an electrical vibration absorber. Further, the advantages and disadvantages, in terms of performance and robustness, of such systems are investigated both theoretically and experimentally.

\section{THEORY}

\section{A. Control Methods}

Consider a single mount vibration isolation system consisting of a rigid equipment mass $m_{e}$ connected to a flexible base structure via a single mount consisting of a spring $k_{m}$ and a damper 


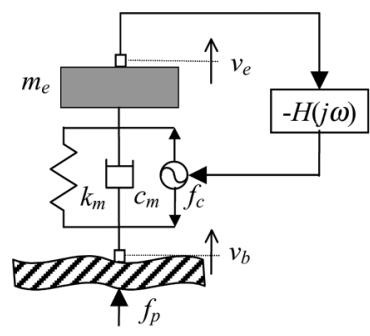

(a)

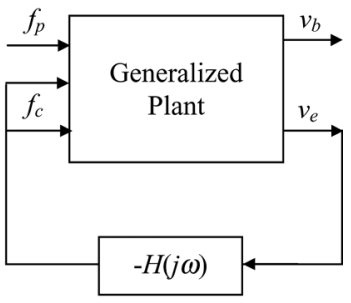

(b)
Fig. 1. Simplified model of (a) a single-mount feedback control system and (b) its block diagram.

$c_{m}$ as shown in Fig. 1(a). A primary force $f_{p}$ is located at the mount position for convenience, and excites the base structure. An electromagnetic actuator generating a pair of control forces $f_{c}$ is also installed in parallel with the passive mount to further reduce vibration transmission from the base velocity $v_{b}$ to the equipment velocity $v_{e}$. The feedback control system uses the equipment velocity $v_{e}$ to generate the control forces $f_{c}$ via the control filter $H(j \omega)$, i.e., $f_{c}=-H(j \omega) v_{e}$. Because of the reaction force acting on the base, it is a two-input-one-output control system as depicted in Fig. 1(b). The dynamic equations of the whole electro-mechanical system can be written in matrix form as [4]

$$
\left[\begin{array}{cc}
Z_{e}+Z_{m} & -Z_{m} \\
-Z_{m} & Z_{b}+Z_{m}
\end{array}\right]\left\{\begin{array}{l}
v_{e} \\
v_{b}
\end{array}\right\}=\left\{\begin{array}{c}
f_{c} \\
f_{p}-f_{c}
\end{array}\right\}
$$

where the impedances of the equipment and mount are $Z_{e}=$ $j \omega m_{e}$ and $Z_{m}=c_{m}+k_{m} / j \omega$ with $\omega$ being the angular frequency; $Z_{b}=1 / Y_{b}$, where $Y_{b}$ is the mobility of the base structure with the equipment and isolator removed. The frequency dependency of all the variables in (1) is omitted for brevity.

From (1), the plant response is given by

$$
G(j \omega)=\frac{v_{e}}{f_{c}}=\frac{Z_{m e}}{1+Z_{m e} Y_{b}} \cdot \frac{1}{Z_{e} Z_{m}}
$$

where $Z_{m e}=Z_{e} Z_{m} /\left(Z_{e}+Z_{m}\right)$ is the impedance of the mounted equipment looking upwards from the bottom of the mount. When the mounted equipment has little effect on the base motion it is said to be weakly coupled and $\left|Z_{m e} Y_{b}\right|<<1$. In this case, the influence of the base dynamics can be ignored and the plant simplifies to the mobility of a single degree-of-freedom (DOF) vibration system such that

$$
G_{o}(j r)=\frac{1}{Z_{e}+Z_{m}}=\frac{1}{c_{m}} \cdot \frac{j a r}{1-r^{2}+j a r}
$$

where $r=\omega / \omega_{m}$ with $\omega_{m}=\sqrt{k_{m} / m_{e}}$ being the natural frequency of the mounted equipment; $a=2 \zeta_{m}$ is the half-power bandwidth; and $\zeta_{m}=c_{m} /\left(2 \sqrt{m_{e} k_{m}}\right)$ is the damping ratio. The weakly coupled plant $G_{o}(j r)$ is the nominal plant with which the control filter is later designed.

Two control strategies are investigated in this paper. The first is the implementation of an electrical damper which is achieved by applying direct velocity feedback ( $D V F B)$ control with a constant gain $h[2]$, so that

$$
H(j \omega)=h .
$$

The second is the implementation of an electrical dynamic absorber, which is achieved by feeding back velocity through a second-order band-pass filter so that

$$
H(j r)=h \cdot \frac{j b \alpha r}{1-\alpha^{2} r^{2}+j b \alpha r}
$$

where $h$ is a gain, and $\alpha=\omega_{m} / \omega_{c}$ with $\omega_{c}$ being the natural frequency of the filter; $b=2 \zeta_{c}$ is the bandwidth of the filter; and $\zeta_{c}$ is the damping ratio. It can be seen that the filter in (5a) has a gain $h$ at $\omega_{c}$ and is approximately equivalent in the pass band to that in (4). The same control effect as that achieved with the filter in (5a) can also be achieved if the equipment acceleration is fed back through a second-order low-pass filter instead, given by [6]

$$
H(j r)=\frac{h}{\omega_{c}} \cdot \frac{b}{1-\alpha^{2} r^{2}+j b \alpha r}
$$

which is called acceleration-position feedback (APF) control in this paper. Because acceleration is usually measured in practice it can be seen that the electrical dynamic absorber can be implemented simply by using a low-pass filter.

\section{B. Analysis of Performance}

Assuming a stable system, the velocity response vector $\left\{\begin{array}{ll}v_{e} & v_{b}\end{array}\right\}^{T}$ can be obtained by premultiplying both sides of (1) by the inverse of the system impedance matrix taking into account the control action, $f_{c}=-H(j \omega) v_{e}$. The performance of the isolation system can be quantified three different ways according to need: 1 ) the velocity of the equipment $v_{e} ; 2$ ) the transmissibility $T$ of the mount; and 3 ) the sensitivity function $S$ for assessing the controller performance.

The first description is readily available from the solution to (1). The second description, the transmissibility, can be determined after some algebraic manipulation of (1) to give

$$
T=\frac{v_{e}}{v_{b}}=\frac{Z_{m}}{Z_{e}+Z_{m}+H} .
$$

Because this is independent of the base dynamics it can give some insight into the control concept. If DVFB control is applied, (4) can be substituted into (6) to reveal that the electrical filter works as a sky-hook damper attached to the equipment [3]. The equivalent skyhook damping ratio is given by $\zeta_{\text {sky }}=g \zeta_{m}$, where $g=h / c_{m}$. If APF control is applied, (5a) can be substituted into (6) to reveal that the filter works as a dynamic absorber attached to the equipment [11]. Although (5a) does not represent any of the mechanical dynamic absorbers considered in [11] or elsewhere, it does represent the impedance of an acoustic cavity resonator [12]. If the filter damping ratio is much less than 1 , however, (5a) has the same form as that of the impedance of a dynamic absorber consisting of a mass and a spring [12]. The equivalent mass ratio between the absorber mass $m_{c}=b h / \omega_{c}$ and the equipment mass $m_{e}=c_{m} /\left(a \omega_{m}\right)$ can thus be determined, and is given by $\sigma=m_{c} / m_{e}=a b g$, where $a=2 \zeta_{m}, b=2 \zeta_{c}$, and $g=h / c_{m}$, provided that $\omega_{c}=\omega_{m}$.

Such analogous systems are exact only if the coupling between the mounted equipment and the base structure is weak. Although this is not strictly true in a vibration isolation system, the interpretation of the two control systems in terms of mechanical analogies is considered to be useful.

Last, the sensitivity function $S$ represents the vibration reduction ratio of the equipment after control, (i.e., $S=v_{e} / v_{e 0}$ ) 
where $v_{e}$ and $v_{e 0}$ are the equipment velocities after and before control, respectively. The velocity $v_{e 0}$ can be obtained by setting $f_{c}=0$ in (1). Since the sensitivity function is given by $S=[1+L(j r)]^{-1}[13]$, where the open-loop frequency response function (FRF) is $L(j r)=G(j r) H(j r)$, the vibration reduction ratio (RR) can be written in decibel form as

$$
R R(\mathrm{~dB})=20 \log _{10}|S|=-20 \log _{10}|1+L(j r)| .
$$

Equation (7) is closely related to the robust stability of the system which is discussed in Section II-C.

\section{Stability Analysis}

The stability of the DVFB control system has been discussed previously using the Nyquist stability criterion [4]. Since the controller is simply a constant gain, the stability is completely determined by the plant response. Due to the additional phase lag introduced by the first-order low-pass filter term $1 /\left(Z_{e} Z_{m}\right)=\left(1 / m_{e}\right)\left[1 /\left(k_{m}+j \omega c_{m}\right)\right]$ in (2), the phase of the complete plant response is within the limits

$$
90^{\circ}>G(j r)>-180^{\circ}
$$

provided that $c_{m}>0$. Although one end of the control actuator is collocated with the error sensor, the plant is not a minimum phase system. However, the control system is always stable in theory and is robust unless an extremely large gain is used with a very highly damped mount installed on a very lightly damped base structure [4].

Now consider the APF control system whose open-loop FRF can be constructed by multiplying (2) and (5a). Since the phase of the filter in (5a) ranges between $90^{\circ}>\angle H(j r)>-90^{\circ}$ for $\zeta_{c}>0$, the system open-loop FRF crosses the negative real axis. Thus, its stability now strongly depends on the control filter in (5a) and there are optimal control parameters to achieve the best performance for a given robustness constraint. A strict robust stability criterion is used in this paper by slightly altering a known model in the literature [13]. A system is defined here to be robustly stable with the robustness degree $l$ if and only if the open-loop FRF $L(j r)$ does not enclose or cross the circle of radius $l$ centered at $(-1,0)$ point, where $0<l<1$, that is

$$
|1+L(j r)| \geq l .
$$

Equivalently, combining (7) and (9a) results in the inequality

$$
R R(\mathrm{~dB}) \geq-20 \log _{10} l .
$$

The open-loop FRFs for the two control strategies and their corresponding vibration reduction ratios are illustrated in Fig. 2(a) and (b), respectively. The plant used for both strategies is the nominal plant $G_{o}(j r)$ given by (3), and the APF controller is tuned to the natural frequency of the nominal plant (i.e., $\omega_{c}=\omega_{m}$ ), (this is the condition henceforth in this paper). The two frequencies $r_{1}$ and $r_{2}$ at which the maxima of the reduction ratio occur in Fig. 2(b), are the two natural frequencies of the combined electro-mechanical system, consisting of both a single DOF mechanical system (the mounted equipment) and another single DOF electrical system (the dynamic absorber). (a)

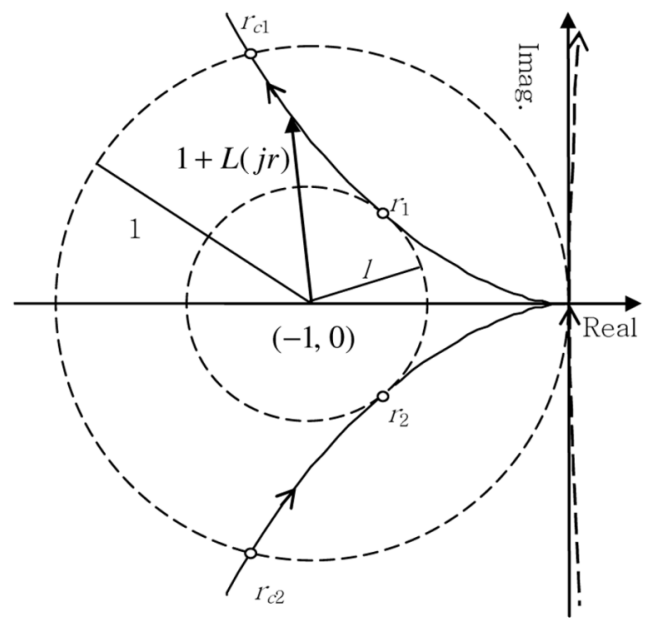

(b)

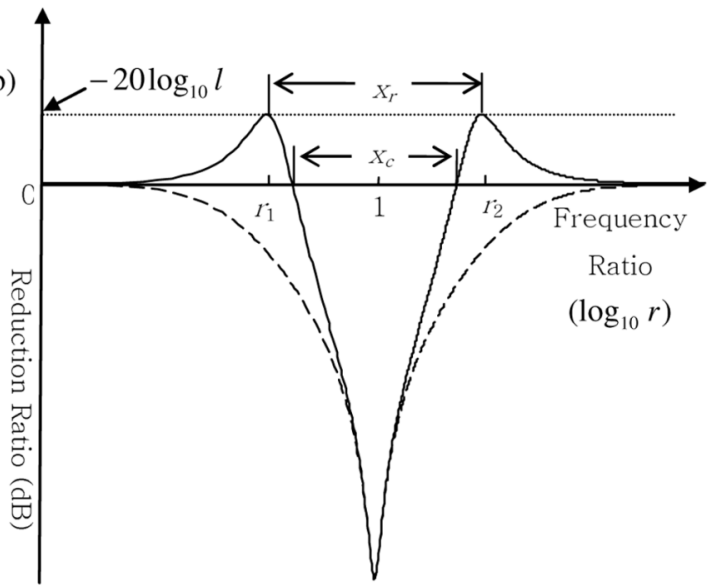

Fig. 2. (a) Open-loop frequency response functions and (b) reduction ratios of DVFB control (dashed lines) and APF control (solid lines) having the robustness degree $l$, for the nominal plant given by (3). The arrows on the curves show the direction of increasing frequency.

The bandwidth between these frequencies is defined as the resonance bandwidth and is denoted as $x_{r}=r_{2}-r_{1}$. The degree of robustness $l$ can also be interpreted in terms of a generalized gain margin by $G M=1 /(1-l)$, where the margin is defined at the resonance frequency giving the minimum distance $l$, not at the phase crossover frequency. The resonance frequencies can be obtained by solving

$$
\frac{d(|1+L(j r)|)}{d r}=0 .
$$

The bandwidth $x_{c}=r_{c 2}-r_{c 1}$, between the two frequencies $r_{c 1}$ and $r_{c 2}$ shown in Fig. 2, is defined as the control bandwidth within which a reduction occurs. This can be obtained from

$$
|1+L(j r)|=1 .
$$

Since the control filter is tuned so that $\omega_{c}=\omega_{m}$, there are two parameters to be determined, namely the gain $h$ and the damping ratio $\zeta_{c}$. Combining (3) and (5a), and introducing a new variable $x=r-r^{-1}$, the open-loop FRF can be written as

$$
L(j x)=g a b(a+j x)^{-1}(b+j x)^{-1} .
$$


Substituting (12) into (10) and differentiating gives

$$
r_{1,2}=\frac{\left(\sqrt{x_{r}^{2}+4} \pm x_{r}\right)}{2}
$$

where $x_{r}=\sqrt{y_{r}}$ is the resonance bandwidth and

$$
y_{r}=\nu\left(1+\frac{g}{2}\right)+\sqrt{\nu^{2}\left(1+\frac{g}{2}\right)^{2}+2 \mu \nu\left(1+\frac{g}{2}\right)+\nu^{2}}
$$

in which $\mu=\left(a^{2}+b^{2}\right) / 2$ and $\nu=a b$. It is interesting to note that $x_{r}=r_{2}-r_{1}$ and $r_{1}=1 / r_{2}$, where $r_{2}>1$. For the control bandwidth, substituting (12) into (11) gives

$$
y_{c}=\nu\left(1+\frac{g}{2}\right) \text {. }
$$

The two corresponding roots $r_{c 1}$ and $r_{c 2}$ can be similarly obtained using the relations $x_{c}=\sqrt{y_{c}}$ and (13).

Now, substituting (12) into (9a) and setting the inequality to an equality gives

$$
|1+L(j r)|^{2}=\frac{y^{2}+2(\mu-\nu g) y+\nu^{2}(1+g)^{2}}{y^{2}+2 \mu y+\nu^{2}}=l^{2}
$$

where $y=x^{2}$ and again $x=r-r^{-1}$. Substituting (14) into (16) gives

$$
\left(C^{2}-1\right) g^{2} v^{2}-4(C+1)(\mu+\nu) g \nu+4\left(\mu^{2}-\nu^{2}\right)=0
$$

where $C=\left(1+l^{2}\right) /\left(1-l^{2}\right)$, which can be used to determine the optimum parameters. If the task is to find the optimal gain $g$ for a given bandwidth $b,(17)$ can be solved to give

$$
g=\left(\frac{(\eta+1)\left(1-l^{2}\right)}{l^{2}}\right)\left(1+\sqrt{1-l^{2}\left(\frac{(\eta-1)}{(\eta+1)}\right)}\right)
$$

where $\eta=\mu / \nu$. On the other hand, if the task is to find the optimal damping ratio for a given target gain $g$, the solution can be written as

$$
\zeta_{c} \approx\left(\frac{g a}{\left(1-l^{2}\right)}\right)\left(1-\sqrt{\left(1-l^{2}\right)\left(1+\frac{2}{g}\right)}\right)
$$

provided that $a^{2}<<b^{2}$. Equations (18) and (19) can be used to determine the optimal parameters for the best performance with a prescribed degree of robustness $l$, as defined in (9).

\section{EXPERIMENTAL WORK}

\section{A. Description of the Control Systems}

A single active mount system similar to that shown in Fig. 1 was experimentally implemented as shown in Fig. 3. The equipment of mass $m_{e}=2.45 \mathrm{~kg}$ containing an electro-magnetic actuator (Ling Dynamic Systems V201: capacity $200 \mathrm{~N}$ and useful range $5-13000 \mathrm{~Hz}$ ) and its fixing frames was installed at the center of a clamped-clamped-free-free steel plate of dimensions $(700 \times 500 \times 3 \mathrm{~mm})$ using a commercial mount (Fabreeka 51505-2) of stiffness $k_{m}=21.1 \times 10^{4} \mathrm{~N} / \mathrm{m}$ and damping $c_{m}=$ $100.7 \mathrm{Ns} / \mathrm{m}$. The mounted equipment had a natural frequency

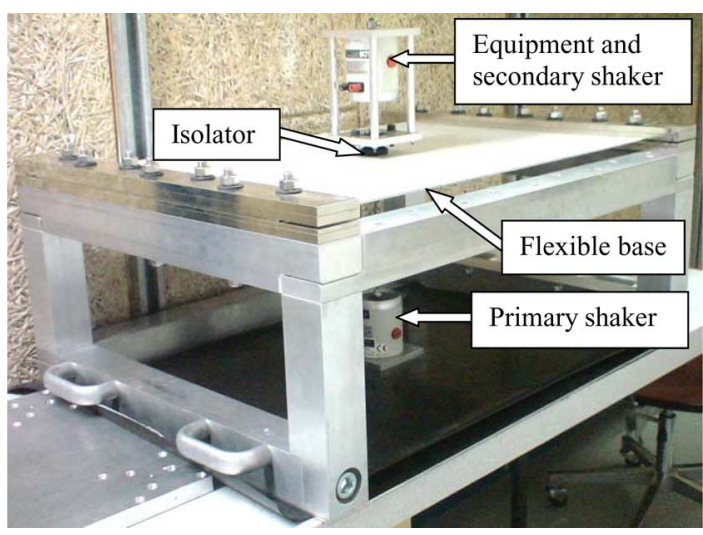

Fig. 3. Piece of equipment containing the electro-magnetic control actuator and its fixing frames is attached to a steel base plate via a passive mount.

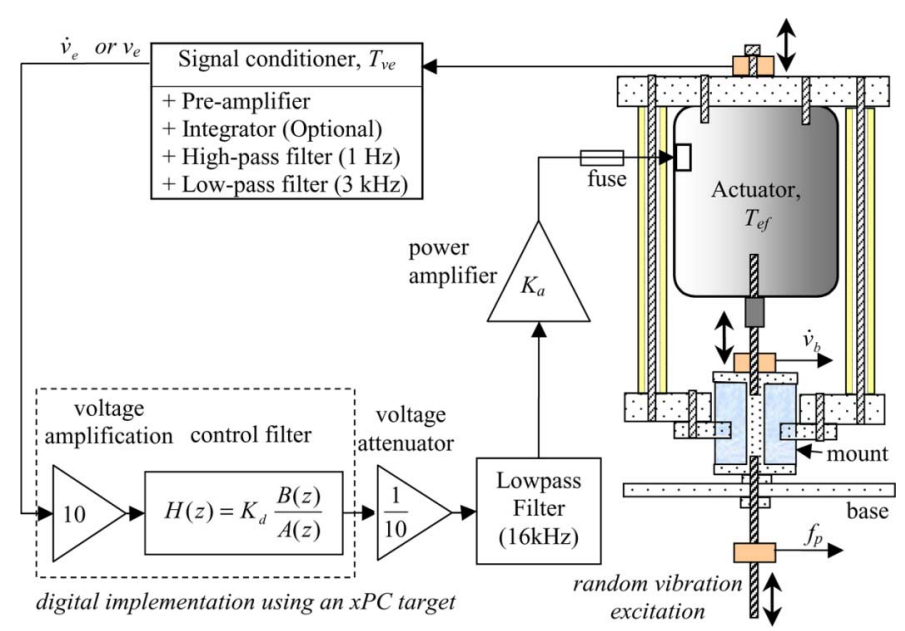

Fig. 4. Structure of the controller and a schematic drawing of the active mount system, where double-sided arrows show the direction of vibration and singlesided arrows indicate signal flows.

of $f_{m}=46.7 \mathrm{~Hz}$ and damping ratio $\zeta_{m}=0.07$. The shorter sides of the rectangular base plate were clamped to a heavy frame made of rectangular aluminum columns of cross sectional dimensions $(60 \times 60 \mathrm{~mm})$. The base plate was excited by another electro-magnetic actuator (LDS V201) underneath the plate. As shown in the schematic drawing in Fig. 4, a stinger was connected between the equipment and the base plate to generate a control force. The equipment response was measured using an accelerometer (Endevco 7251A-10), and the base response and the primary force were monitored by another accelerometer of the same type and a force transducer (PCB 208C03), respectively. Although not shown in Fig. 4, there was a spectrum analyzer (LMS CADA-X) used for generating a random signal supplied to the primary actuator as well as monitoring the primary excitation force $f_{p}$ and the motions of the base $\dot{v}_{b}$ and the equipment $\dot{v}_{e}$.

Fig. 4 also shows the signal flow in the experimental setup. The accelerometer output from the equipment passed through a signal conditioner (Nexus) equipped with a mandatory band-pass filter with an optional integrator to obtain a velocity response for DVFB control. The integrator was bypassed for APF control. In all control cases, the pass bandwidth was set to be $1 \mathrm{~Hz} \leq f \leq 3 \mathrm{kHz}$. As denoted inside a dashed 
block, the control filters were implemented digitally using a Matlab xPC target processor (National Instruments PCI-6040E combined with BNC-2090 as a data acquisition front-end). The digital processor was used so that the filter parameters could be changed easily. The discrete form for DVFB control is the same as its analog form in (4). The discrete form for the APF control filter for a gain $K_{d}$ can be determined by applying the impulse invariant transform method, and is given by [14]

$$
H(z)=K_{d} \frac{2 \zeta_{c} \omega_{c} T}{\omega_{d}} \cdot \frac{z A \sin \omega_{d} T}{z^{2}-z\left(2 A \cos \omega_{d} T\right)+A^{2}}
$$

where $\omega_{d}=\omega_{c} \sqrt{1-\zeta_{c}^{2}}, A=\exp \left(-\zeta_{c} \omega_{c} T\right)$, and the sampling time is $T=1 / F_{s}$ where $F_{s}$ is the sampling frequency. With the high sampling frequency of $32 \mathrm{kHz}$ used, (20) closely resembles its analog counterpart, given by (5b), below the Nyquist frequency. A low-pass filter (Rockland 432) with cutoff frequency $16 \mathrm{kHz}$ was also used to reconstruct an analog signal. The cutoff frequency was set sufficiently high to avoid a large phase delay in the relatively low frequency range of interest. Finally, the filtered signal was amplified by an audio power amplifier (Hafler P1500 with a dynamic range of $15 \mathrm{~dB}$ ) of gain $K_{a}$ and transmitted to the actuator through a safety fuse $(250 \mathrm{~V}, 2 \mathrm{~A})$ that was used to protect the actuator from saturation in case of instability. If all the transducers and signal conditioning electronic devices used perform ideally, the normalized controller gain $g$ for DVFB control can be written as

$$
g=\frac{K T_{v e} T_{e f}}{c_{m}}
$$

where $K=K_{d} K_{a}$ in which $K_{d}$ and $K_{a}$ are the gains used in the digital and analog systems, respectively, $T_{v e}$ is the transformation factor from velocity to voltage in the signal conditioner and $T_{e f}$ is the transformation factor from voltage to force in the electromagnetic actuator. The gain $g$ for APF control can be similarly defined using an appropriate transformation factor from acceleration to voltage, instead of $T_{v e}$.

The plant response $G(j \omega)=v_{e} / f_{c}$ was measured using the input to the power amplifier and the output from the signal conditioner in Fig. 4. The measurement was conducted both on the flexible base and a rigid base to obtain the coupled and uncoupled plant responses, respectively. The measured coupled (solid lines) and uncoupled (dashed lines) plants are compared in Fig. 5 for the frequency band $2.5 \mathrm{~Hz} \leq f \leq 1.8 \mathrm{kHz}$. The responses less than $2.5 \mathrm{~Hz}$ were very noisy due to low sensitivity in the actuator and the mechanical plant. The frequency and amplitude of the small peak at $33 \mathrm{~Hz}$ (solid line) were found to be highly dependent on the location of a supporting leg that was placed under the table (directly below the primary actuator position) to adjust the table stiffness. This peak could possibly be due to strong coupling between the heavy experimental system (including the mounted equipment and the base frame) and the ground (the flexible supporting table). At frequencies above $1.5 \mathrm{kHz}$, a number of resonances are present. The largest peak at $1590 \mathrm{~Hz}$ is a higher-order resonance of the mechanical structure containing the equipment and mount. It should be noted that the upper cutoff frequency of the signal conditioner had been purposely set to $3 \mathrm{kHz}$ in order to show

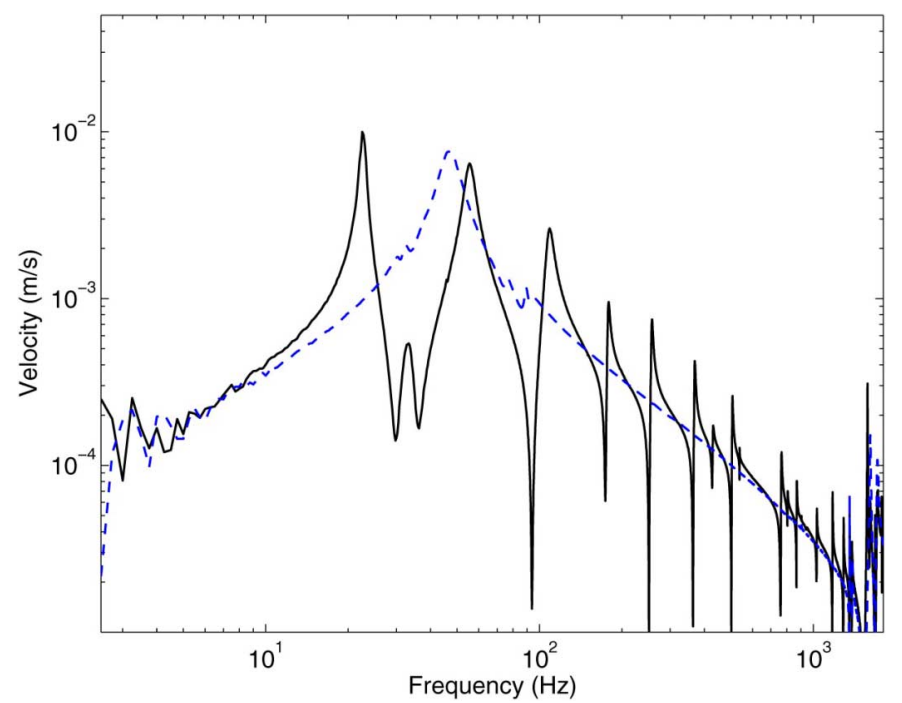

Fig. 5. Measured coupled (solid line) and uncoupled (dashed line) plant responses to the control force $1 \mathrm{~N}$.

this sort of unmodeled effect of the electro-mechanical plant. In Fig. 5, it is striking that the coupled plant is asymptotically similar to the uncoupled plant. Close examination of the criterion for weak coupling, $\left|Z_{m e} Y_{b}\right|<<1$, reveals that $\left|Z_{m e}\right|$ indeed becomes relatively small at frequencies away from the natural frequency $\omega_{m}$. This means the uncoupled plant (the mounted equipment) can be used to determine the optimal filter parameters for the coupled plant. The accuracy depends on various factors, for example, the control bandwidth chosen, the high frequency damping behavior of the base, higher order modes of the mounted equipment, etc.

The amplitudes and complex loci of the open-loop FRFs of DVFB (dashed lines) and APF (solid lines) control are shown in Fig. 6(a) and (b), respectively. They were measured using a random signal input to the $\mathrm{xPC}$ target and the output from the signal conditioner in Fig. 4. The gain used was $g=15.5$ and the optimal damping ratio was calculated from (19), setting the robustness degree $l=1 / \sqrt{2}$, which corresponds to a maximum allowable amplification of about $3 \mathrm{~dB}$ after control and a generalized gain margin $(\mathrm{GM})$ of $10.7 \mathrm{~dB}$. The prescribed robustness degree is represented in Fig. 6(b) by a circle (dotted). It is shown in Fig. 6(a) that both amplitudes are very similar in a bandwidth centered at $f_{m}=46.7 \mathrm{~Hz}$ and the response for APF control diminishes more rapidly at frequencies far away from this, implying that there is a smaller control effort at very low and very high frequencies. In Fig. 6(b), the locus (dashed line) for DVFB control crosses the imaginary axis and enters the left-half plane both at very low and very high frequencies. The low frequency crossing was due to phase advances of the power amplifier and the integrator combined with the high-pass filter within the signal conditioner used. The high frequency crossings were due to phase delays of the mechanical plant, the electromagnetic actuator, and the low-pass filters used. These crossings could make the system unstable for a large gain. A detailed discussion on these issues is presented in the Appendix. The response (solid line) for APF control crosses the robust stability boundary $l=1 / \sqrt{2}$ at high frequencies. However, the maximum intrusion into the circle is only by about $4.4 \mathrm{~dB}$, which 


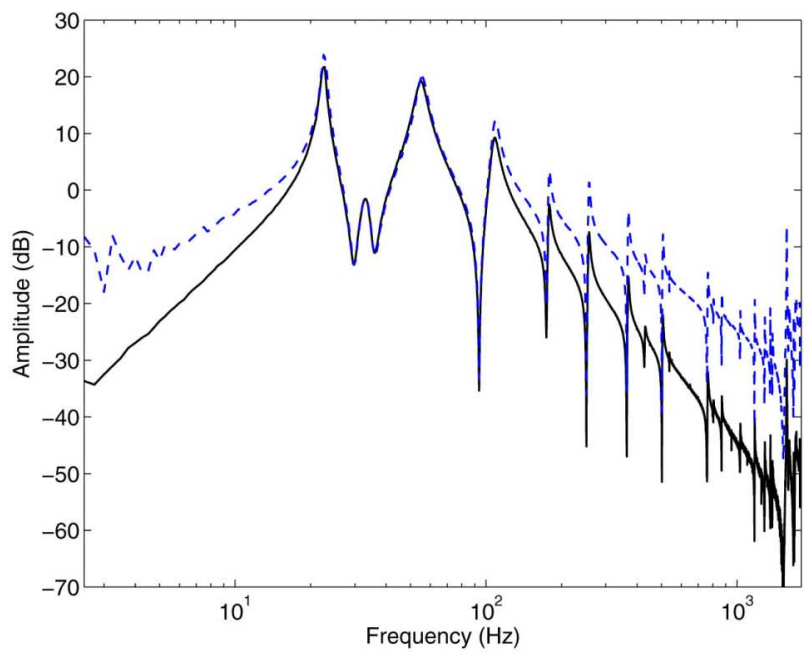

(a)

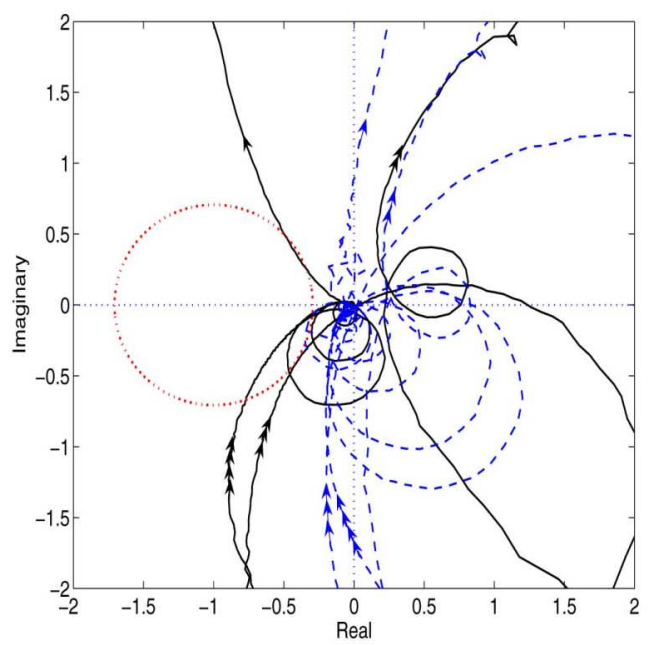

(b)

Fig. 6. Measured open-loop FRFs of DVFB (dashed lines) and APF (solid lines) control of robustness degree represented by a circle of radius $l$ in (b). The arrows in (b) indicate the direction of increasing frequency starting from a single arrow. (a) Amplitude. (b) Complex domain.

corresponds to a less strict robustness degree of about $l=1 / 2$ and $G M=6 \mathrm{~dB}$.

\section{B. Control Performance and Robust Stability}

Isolation systems with five normalized gains of $g=0,5.2$, $15.5,27$, and 54 were tested. The case $g=0$ corresponds to the original passive system before control. For each gain, DVFB and APF control were tested successively. The input signal to the primary shaker was a random signal for all cases. The optimal damping ratio of the APF controller was obtained from (19) with a robustness degree $l=1 / \sqrt{2}$. The filter parameters and properties are given in Table I. Properties of the analogous electrical damper and dynamic absorber are also summarized in Table I. The active system was stable for all gains tested, and no further fine tuning of the controller damping ratio was conducted. Using electrical means, therefore, it was possible to achieve an equivalent skyhook damping ratio of 3.78 and an equivalent absorber mass ratio as high as 64 .

Fig. 7(a) shows the predicted and measured equipment velocities of DVFB control for the four gains used, where the original
TABLE I

Control Filter Parameters AND Nominal Properties of the ACtive Mount System HaVING a PASSIVE DamPING RATIO OF 0.07

\begin{tabular}{l|cccc}
\hline Normalized gain, $g$ & 5.2 & 15.5 & 27 & 54 \\
\hline Controller damping ratio, $\zeta_{c}$ & 0.24 & 1.08 & 2.02 & 4.23 \\
Skyhook damping ratio, $\zeta_{s k y}$ & 0.36 & 1.08 & 1.89 & 3.78 \\
Absorber mass ratio, $\sigma$ & 0.4 & 4.7 & 15.2 & 63.9 \\
Nominal max. reduction (dB) & 15.8 & 24.3 & 28.9 & 34.8 \\
Pass bandwidth, $b$ & 0.49 & 2.15 & 4.04 & 8.46 \\
{$\left[\omega_{p 1} \sim \omega_{p 2}\right](\mathrm{Hz})$} & {$[36.7 \sim 59.4]$} & {$[18.3 \sim 118.9]$} & {$[10.9 \sim 199.4]$} & {$[5.4 \sim 400.4]$} \\
Control bandwidth, $x_{c}$ & 0.49 & 1.62 & 2.86 & 5.76 \\
{$\left[\omega_{c 1} \sim \omega_{c 2}\right](\mathrm{Hz})$} & {$[36.6 \sim 59.7]$} & {$[22.2 \sim 98.0]$} & {$[14.7 \sim 148.3]$} & {$[7.9 \sim 276.7]$} \\
Resonance bandwidth, $x_{r}$ & 0.78 & 2.65 & 4.73 & 9.60 \\
{$\left[\omega_{r 1} \sim \omega_{r 2}\right](\mathrm{Hz})$} & {$[32.0 \sim 68.2]$} & {$[15.6 \sim 139.4]$} & {$[9.5 \sim 230.3]$} & {$[4.8 \sim 452.9]$} \\
\hline
\end{tabular}

where $\zeta_{s k y}=g \zeta_{m}, \sigma=m_{c} / m_{e}=a b g$ with $a=2 \zeta_{m}$ and $b=2 \zeta_{c}$, the maximum reduction of the nominal plant is $-20 \log _{10}(1+g), \zeta_{c}$ is from (19), and $x_{r}$ and $x_{c}$ are from (14) and (15), respectively.

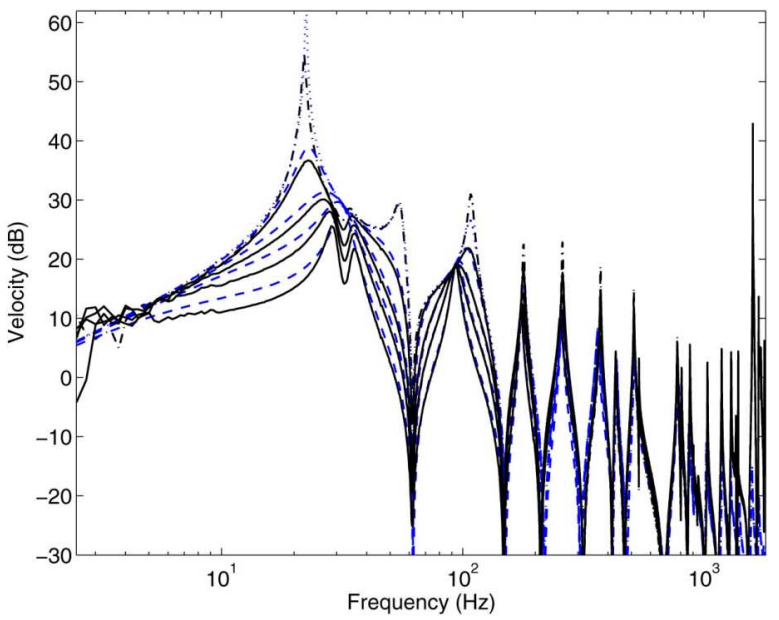

(a)

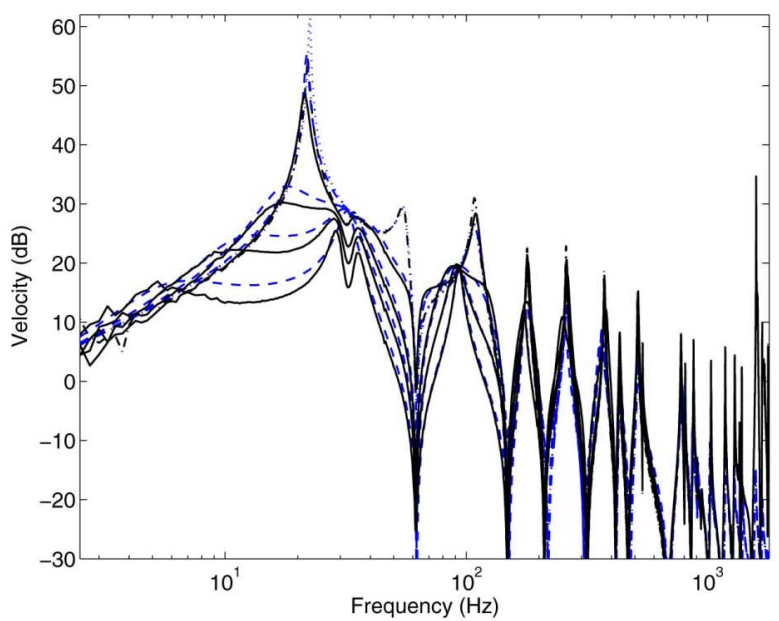

(b)

Fig. 7. Predicted (dashed lines) and measured (solid lines) velocity responses of the equipment for various gains of (a) DVFB and (b) APF control are compared with the original velocity responses before control (predicted: dotted lines, measured: dashed-dotted lines). Those four decreasing responses, after control (solid and dashed lines), at the first resonance frequency correspond to the four increasing steps of the gain $g=5.2,15.5,27$, and 54 .

responses before control are also shown for comparison. Responses less than $2.5 \mathrm{~Hz}$ are again excluded in the plots. There is good agreement between the predicted and measured results for 
TABLE II

Control Performance of the Active Mount System

\begin{tabular}{l|llllll}
\hline \multicolumn{2}{l}{ Normalized gain, $g$} & & 5.2 & 15.5 & 27 & 54 \\
\hline Maximum & Theory & DVFB & 22.7 & 31.8 & 36.6 & 42.6 \\
& & APF & 13.3 & 30.1 & 36.0 & 42.4 \\
\cline { 2 - 7 } $\begin{array}{l}\text { reduction } \\
\text { (dB) }\end{array}$ & \multirow{2}{*}{ Exp. } & DVFB & 18.6 & 27.5 & 32.4 & 38.4 \\
& & APF & 12.9 & 25.5 & 31.7 & 38.3 \\
\hline \multirow{2}{*}{$\begin{array}{l}\text { Overall } \\
\text { reduction }\end{array}$} & \multirow{2}{*}{ Theory } & DVFB & 11.9 & 16.3 & 18.7 & 21.7 \\
\cline { 2 - 7 } (dB) & \multirow{2}{*}{ Exp. } & DPF & 4.0 & 14.6 & 18.0 & 21.4 \\
& & APF & 2.8 & 11.2 & 14.6 & 18.3
\end{tabular}

The overall reductions were calculated from the responses between $2.5 \mathrm{~Hz}$ $\leq f \leq 1.5 \mathrm{kHz}$.

all gains used, and as expected the velocity decreases monotonically as the gain increases. Slight discrepancies at frequencies less than about $20 \mathrm{~Hz}$ are due to phase advances of the signal conditioning devices used, which were not accounted for in the theoretical model. Discrepancies at very high frequencies above about $1 \mathrm{kHz}$ are mainly due to un-modeled high-order modal behavior of the mounted equipment, as discussed in the previous section. The corresponding results for APF control are shown in Fig. 7(b). Again, there is good agreement between the predicted and measured results. The reasons for slight discrepancies are similar to those for the DVFB control. It can be seen that performances for both controllers are broadly similar.

The maximum reductions for various gains are tabulated in Table II. It is observed that both DVFB and APF controllers give very similar reductions. For the largest gain, a maximum reduction of about $38 \mathrm{~dB}$ is achieved at the first resonance frequency for both controllers. The overall reductions for each control system are also tabulated in Table II, where the calculations were made in the frequency range $2.5 \mathrm{~Hz} \leq f \leq$ $1.5 \mathrm{kHz}$ to avoid the very low frequency noisy data and the very high frequency undesirable dynamics. The maximum overall reductions achieved are 19.2 dB for DVFB control and $18.3 \mathrm{~dB}$ for APF control. This demonstrates that electrical dynamic absorbers work as well as electrical dampers for active vibration isolation. The theoretical and measured system transmissabilities are shown in Fig. 8(a) and (b) for DVFB and APF control, respectively. Again, the measured and predicted results agree in the low- and mid-frequency regions. As the gain increases, it is seen that the resonance bandwidth increases. The level difference between the predicted and measured peaks for each gain is due to the unmodeled phase shifts as discussed earlier.

To examine the amplification characteristics of the experimental system at high frequencies, the vibration reduction ratios of both DVFB and APF control are shown in Fig. 9(a) for a gain $g=27$ and the corresponding equipment velocities already shown in Fig. 7 are redrawn in Fig. 9(b) together with the original response (dashed-dotted line) before control. In Fig. 9(a), the level of $3 \mathrm{~dB}$ (dotted line) is shown for comparison. In Fig. 9(a) and (b), the velocity amplitudes of the higher order mode of the mounted equipment at around $1590 \mathrm{~Hz}$ are indicated by " $\mathrm{x}$ " for DVFB control and " $\mathrm{O}$ " for APF control for clear comparison. As illustrated in Fig. 2(b), DVFB control is

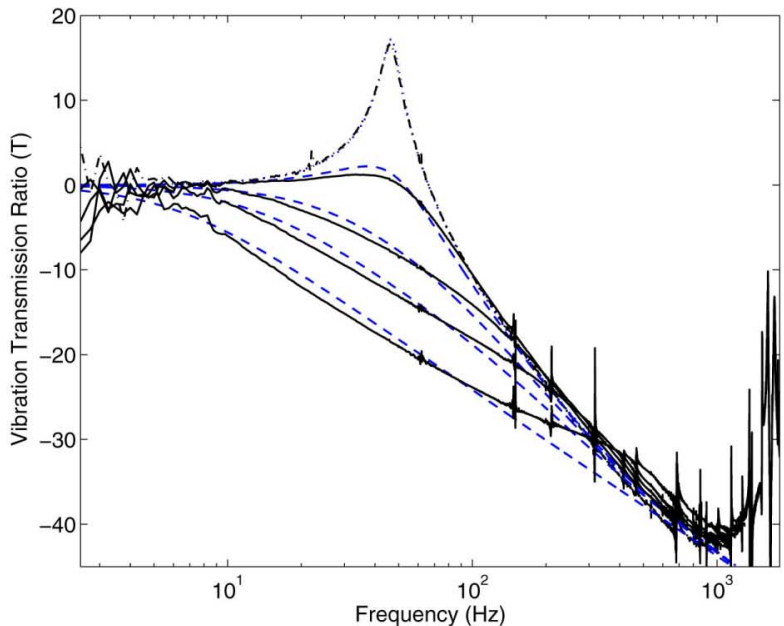

(a)

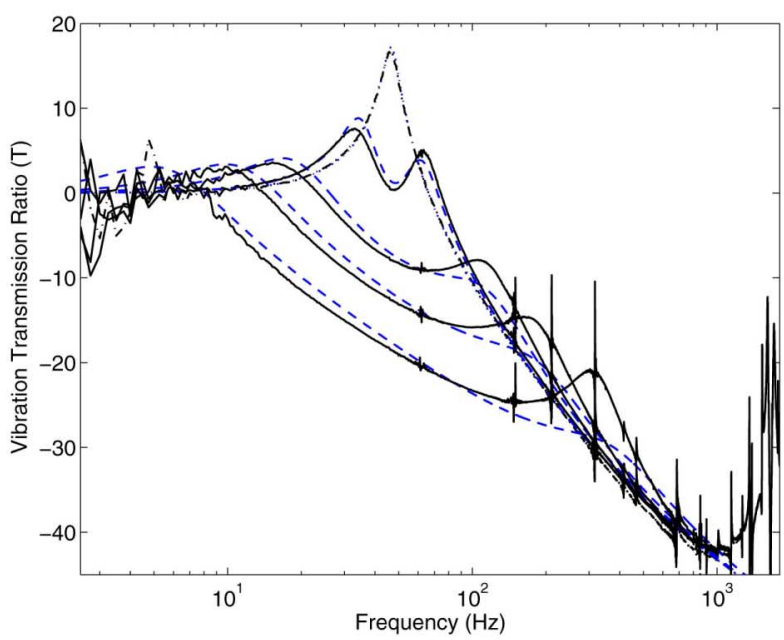

(b)

Fig. 8. Predicted (dashed lines) and measured (solid lines) vibration transmission ratios of the active mount for various gains of (a) DVFB and (b) APF control are compared with those for the passive mount (predicted: dotted lines, measured: dashed-dotted lines). The four decreasing responses, after control (solid and dashed lines), at the first resonance frequency correspond to the four increasing steps of the gain $g=5.2,15.5,27$, and 54 .

theoretically always better than APF control in terms of both performance and stability at all frequencies. However, the experimental results in Fig. 9 show that this is not the case in practice. The vibration reduction ratio for DVFB control (dashed line) in the high frequency region above about $500 \mathrm{~Hz}$ turns out to be larger than that for APF control (solid line). At the higher order resonance frequency, it should be noticed that DVFB control amplifies the equipment motion by as large as about $25 \mathrm{~dB}$ (" $\mathrm{x}$ " in Fig. 9), while no significant amplification is observed for APF control. The same trend could happen at very low frequencies below $2.5 \mathrm{~Hz}$ now with a possible ground resonance mode together with phase shifts introduced by the transducers and the electronics used for signal conditioning [4]. It is important for robustness that the controller is inactive at very low and very high frequencies where undesirable dynamics arise. Indeed, the electrical dynamic absorber (APF control) better suits this requirement as demonstrated in Fig. 6(a). Fig. 9 supports the argument that, for the specific case considered, the electrical dynamic absorber is more robust than the electrical damper. 


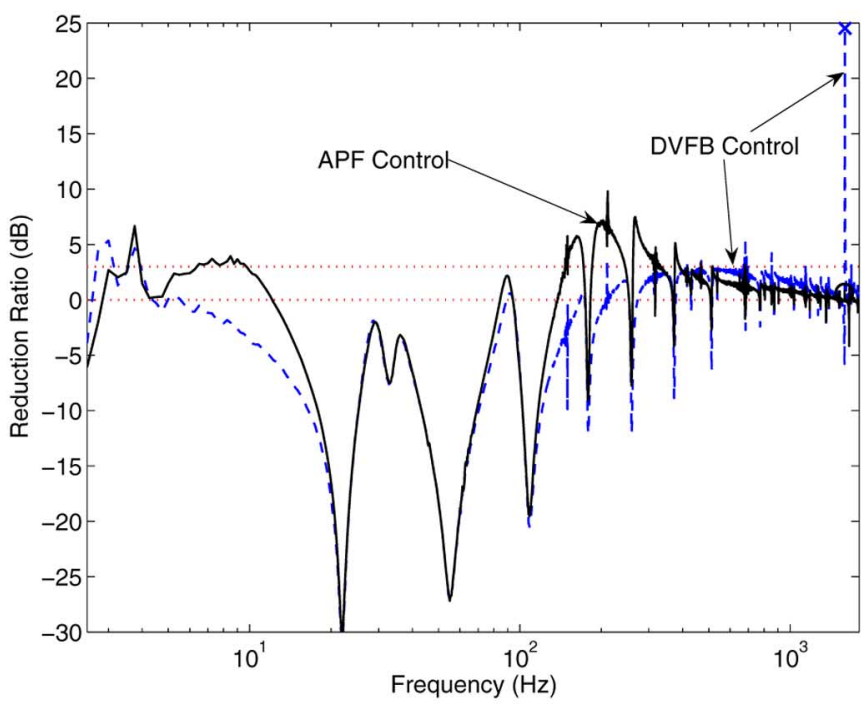

(a)

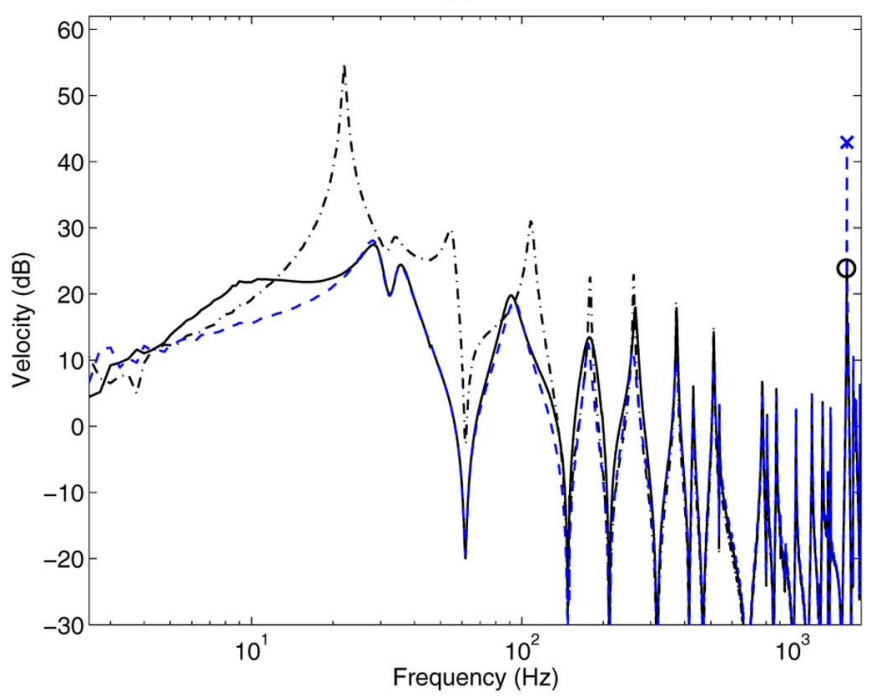

(b)

Fig. 9. Measured reduction ratios of the DVFB (dashed line) and APF (solid line) controllers having the gain 27 together with the level of $3 \mathrm{~dB}$ (dotted line) (a) and the corresponding equipment velocities together with the original velocity (dashed-dotted line) before control. The velocity amplitudes of the higher order mode of the mounted equipment at around $1590 \mathrm{~Hz}$ are indicated by " $\mathrm{x}$ " for DVFB control and "o" for APF control.

\section{CONCLUSION}

In this paper, active vibration isolation systems have been investigated both theoretically and experimentally. Two control strategies have been studied: DVFB control, which effectively adds a skyhook damper to the system, and APF control, which effectively adds a vibration absorber to the system.

Based on the fact that the plant on a flexible base is asymptotically similar to that on a rigid base, the optimal filter parameters were determined for APF control independent of the base dynamics. Thus, only a single filter was used for the active mount and its natural frequency was tuned to that of the mounted equipment on the rigid ground. A robust optimal control framework has been presented that determines the optimal gain and damping ratio for the controller to achieve the best performance with a prescribed robustness. Experimental results demonstrate that it is possible to realize an electrical dynamic absorber that works as well as an electrical damper in terms of performance. A maximum reduction of about $38 \mathrm{~dB}$ was achieved in practice for both controllers. The overall reductions in the frequency band $2.5 \mathrm{~Hz} \leq f \leq 1.5 \mathrm{kHz}$ of 19 and $18 \mathrm{~dB}$ were achieved for DVFB and APF control, respectively.

It was demonstrated that it is possible to realize an electrical dynamic absorber that is more robust than an electrical damper in terms of robustness to undesirable dynamics outside the control bandwidth. This is because an electrical dynamic absorber generates a smaller control effort at very low and very high frequencies. Another important advantage of an electrical dynamic absorber is that, because it can be implemented using an acceleration response, the control system does not require an integrator to transform acceleration into velocity.

\section{APPENDIX \\ UnMODELED DyNAMICS OF THE PRACTICAL CONTROL SYSTEM}

In the theoretical model, it is assumed that the transducers and signal conditioning electronic devices in Fig. 4 are ideal as described in (21). In practice, some distortion is unavoidable. If control filters are assumed to be implemented using analog circuits and velocity is used for feedback, the final distortion in the feedback loop may be represented as

$$
\Gamma(j \omega)=P A(j \omega) H P(j \omega) I(j \omega) L P(j \omega) A C T(j \omega)
$$

where $P A(j \omega)$ denotes the distortion from the audio power amplifier, $H P(j \omega) I(j \omega) L P(j \omega)$ is that from the signal conditioner containing mandatory high- and low-pass filters, $H P(j \omega)$ and $L P(j \omega)$, and an optional integrator $I(j \omega)$, and $A C T(j \omega)$ is that from the electromagnetic actuator.

Each distortion term was separately measured and then mathematically modeled. Each measurement was a FRF between input and output voltages. The distortion of the integrator was obtained by dividing the measured FRF by the ideal integrator $1 / j \omega$. The actuator distortion was measured using a force transducer connected to the stinger when the mounted equipment was installed on a rigid base. Distortions $P A(j \omega)$ and $H P(j \omega)$ were modeled using first-order high-pass filters with cutoff frequencies, $f_{o}, 0.13$ and $1 \mathrm{~Hz}$, respectively. The distortion from the integrator $I(j \omega)$ was modeled using a second-order highpass filter with a cutoff frequency $1 \mathrm{~Hz}$ and the damping ratio 0.7. $L P(j \omega)$ was modeled using a pure time delay of $56 \mu \mathrm{s}$. $A C T(j \omega)$ was modeled using a first-order low-pass filter of cutoff frequency $f_{o}=R_{o} /\left(2 \pi L_{o}\right)=1030 \mathrm{~Hz}$, where $R_{o}$ and $L_{o}$ are, respectively, the resistance and inductance of the electric circuit inside the electromagnetic actuator [15]. This model is quite accurate at low frequencies, provided the actuator is subjected to a large mechanical load having a high impedance [4], as the one considered in this paper.

The measured (solid lines) and identified (dashed lines) amplitude and phase distortions are compared in Fig. 10(a) and (b), respectively. It is seen that the two models agree well. The slight distortions of the experimental curves at $47.5 \mathrm{~Hz}$ were due to the resonance of the mounted equipment on the rigid ground. The 


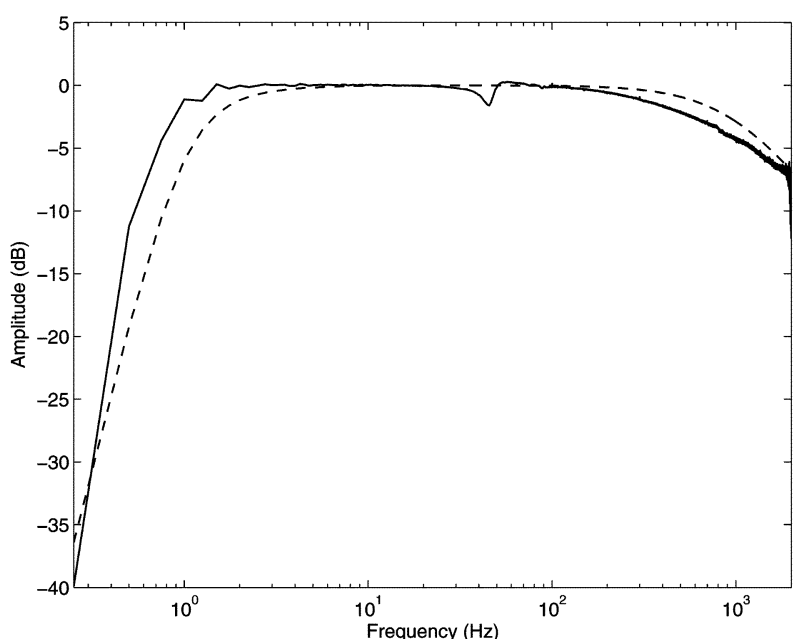

(a)

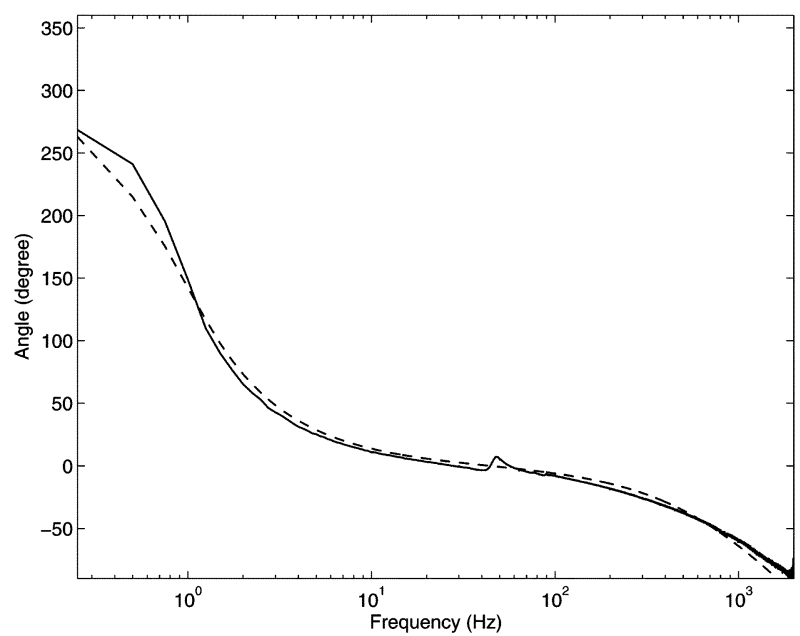

(b)

Fig. 10. Measured (solid lines) and identified (dashed lines): (a) amplitude and (b) phase of the final distortion in (A.1) from a combined system consisting of the power amplifier, the signal conditioner, and the electromagnetic actuator in Fig. 4.

attenuation and phase delay at very high frequencies are mainly due to the low-pass filtering by $A C T(j \omega)$. If one defines the distortion as the deviation from a minimum phase system, there is another important source of distortions in the control loop that has not been considered in (A.1). The mechanical plant in (2) contains a first-order low-pass filter that has an even lower cutoff frequency $f_{o}=k_{m} /\left(2 \pi c_{m}\right)=333.6 \mathrm{~Hz}$. In order to extend the working frequency range of an active mount, the mount damping must therefore be small. This will bring an additional benefit of an improved high frequency vibration isolation performance. The side effect of an increased low frequency vibration transmission could be effectively reduced by active means.

Now consider the low frequency distortion. The low frequency responses in Figs. 10(a) and (b) are typical of a high-pass filter. Notice that the phase at around $1.5 \mathrm{~Hz}$ is advanced by about $90^{\circ}$ but the amplitude is not well attenuated. Theoretically, a phase advance of greater than $90^{\circ}$ at very low frequencies can cause the open-loop FRF locus of a DVFB controller to cross the negative real axis. Such a low frequency phase advance has been known to be a serious threat to the stability of DVFB control [4], [16]. For the particular system considered in this paper, the vibration component at around $1.5 \mathrm{~Hz}$ had the potential to destabilize the system. APF control introduces even larger phase advances at very low frequencies but it also attenuates the modulus of the open-loop FRF locus and so is not destabilizing. It should be noted that for APF control, the distortion $I(j \omega)$ must be excluded from (A.1). In addition, the low-pass filter $L P(j \omega)$ can also be excluded from (A.1). Thus, the signal conditioning electronics can be greatly simplified.

\section{REFERENCES}

[1] D. J. Mead, Passive Vibration Control. New York: Wiley, 1999, pp. 267-313.

[2] M. J. Balas, "Direct velocity feedback control of large space structures," J. Guidance Control, vol. 2, pp. 252-253, 1979.

[3] D. Karnopp, M. J. Crosby, and R. A. Harwood, "Vibration control using the semi-active force generators," J. Eng. Ind., vol. 96, pp. 619-626, 1974.

[4] S. M. Kim, S. J. Elliott, and M. J. Brennan, "Decentralized control for multichannel active vibration isolation," IEEE Trans. Control Syst. Technol., vol. 9, no. 1, pp. 93-100, Jan. 2001.

[5] S. M. Kim, S. J. Elliott, and M. J. Brennan, "Active vibration isolation of a 3-D structure using velocity feedback control," Univ. Southampton, Southampton, U.K., ISVR Tech. Memo. 845, 1999.

[6] E. Sim and S. W. Lee, "Active vibration control of flexible structures with acceleration feedback," J. Guidance Control, vol. 16, pp. 413-415, 1993.

[7] A. Preumont, N. Loix, D. Malaise, and O. Lecrenier, "Active damping of optical test benches with acceleration feedback," Mach. Vibr., vol. 2, pp. 119-124, 1993.

[8] N. W. Hagood and A. von Flotow, "Damping of structural vibrations with piezoelectric materials and passive electrical networks," J. Sound Vibr., vol. 146, pp. 243-268, 1991.

[9] A. J. Fleming, S. O. R. Moheimani, and S. Behrens, "Synthesis and implementation of sensor-less active shunt controllers for electromagnetically actuated systems," IEEE Trans. Control Syst. Technol., vol. 13, no. 2, pp. 246-261, Mar. 2005.

[10] J. L. Fanson and T. K. Caughey, "Positive position feedback control for large space structures," AIAA J., vol. 28, pp. 717-724, 1990.

[11] J. C. Snowdon, "Steady-state behavior of the dynamic absorber," $J$. Acoust. Soc. Amer., vol. 31, pp. 1096-1103, 1959.

[12] S. M. Kim and M. J. Brennan, "Modeling a structural-acoustic coupled system with an equivalent lumped parameter mechanical system," $J$. Vibr. Acoust.- Trans. ASME, vol. 121, pp. 453-459, 1999.

[13] M. Morari and E. Zafiriou, Robust Process Control. Englewood Cliffs, NJ: Prentice-Hall, 1989, pp. 23-36.

[14] S. M. Kim, "Active control of sound in structural-acoustic coupled systems," Ph.D. dissertation, Inst. Sound Vibration Res., Univ. Southampton, Southampton, U.K., 1998.

[15] L. E. Kinsler, A. R. Frey, A. B. Coppens, and J. V. Danders, Fundamentals of Acoustics, 4th ed. New York: Wiley, 2000, pp. 390-411.

[16] M. J. Brennan, K. A. Ananthaganeshan, and S. J. Elliott, "Instabilities due to phase lead and phase lag in feedback control of a single-degree-of-freedom vibrating system," J. Sound Vibr., vol. 304, no. 3-5, pp. 466-478, 2007

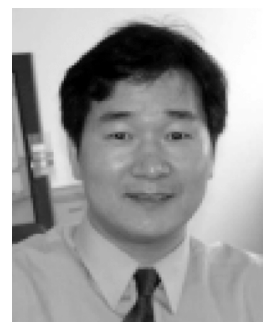

Sang-Myeong Kim received the M.Sc. degree in a study on car interior noise from Hanyang University, Seoul, South Korea, in 1992 and the Ph.D. degree with a thesis entitled "Active control of sound in structural-acoustic coupled systems" from the Institute of Sound and Vibration Research, University of Southampton, Southampton, U.K., in 1998.

$\mathrm{He}$ is currently with Phonak Hearing Systems, Staefa, Switzerland. Prior to this, he worked as a Research Fellow on smart structures with the University of Southampton, an Assistant Professor with Gwangiu Institute of Science and Technology, Korea, and a Senior Scientist with EMPA. His research interests focus on exploring the impedance and Wiener filter methods applied to various areas covering acoustics, vibration, electrodynamics of sensors and actuators, hearing instruments, virtual sound, and active control 


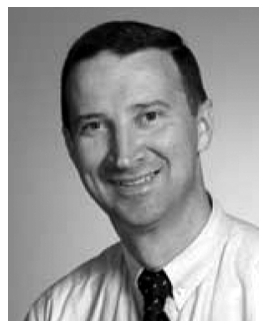

Stanislaw Pietrzko received the M.S. degree with honors in electrical engineering and automatic control from AGH University of Science and Techology, Cracow, Poland, and the Ph.D. degree in mechatronics from ETH Zurich, Zurich, Switzerland, in 1992.

He is currently the Head of the Vibroacoustics Group, EMPA Swiss Federal Laboratories for Materials Testing and Research in Dübendorf, where his research is focused on vibroacoustics, passive and active control of noise vibration, and damping phenomena. He has spent sabbaticals with the Institute of Sound and Vibration Research, Southampton, U.K., and with the University of Adelaide, Adelaide, Australia. He has published numerous papers on noise and vibration control, given invited lectures and short courses on these subjects, and teaches and supervises students.

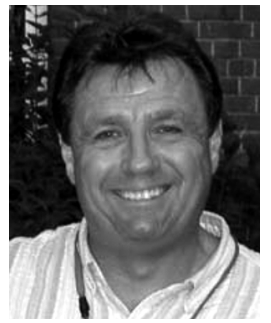

Michael J. Brennan graduated from the Open University in 1987 while he was serving in the Royal Navy. He received the M.Sc. degree in sound and vibration studies and the Ph.D. degree in the active control of vibration from the University of Southampton, Southampton, U.K., in 1992 and 1995, respectively.

$\mathrm{He}$ is currently chairman of the Dynamics Research Group with the Institute of Sound and Vibration Research, University of Southampton, and holds a personal chair in Engineering Dynamics. He has a wide-range of research interests, encompassing vibration, acoustics, vibroacoustics, and rotor dynamics. 